Florida International University FIU Digital Commons

6-14-2012

\title{
New Jellium Model for Alkali Metals and its Future Applications to Metal Clusters
}

Guillermo Matranca

Florida International University, gmatr001@fiu.edu

DOI: $10.25148 /$ etd.FI12071112

Follow this and additional works at: https://digitalcommons.fiu.edu/etd

\section{Recommended Citation}

Matranca, Guillermo, "New Jellium Model for Alkali Metals and its Future Applications to Metal Clusters" (2012). FIU Electronic Theses and Dissertations. 657.

https://digitalcommons.fiu.edu/etd/657

This work is brought to you for free and open access by the University Graduate School at FIU Digital Commons. It has been accepted for inclusion in FIU Electronic Theses and Dissertations by an authorized administrator of FIU Digital Commons. For more information, please contact dcc@fiu.edu. 


\title{
FLORIDA INTERNATIONAL UNIVERSITY
}

Miami, Florida

NEW JELLIUM MODEL FOR ALKALI METALS AND ITS FUTURE APPLICATIONS

TO METAL CLUSTERS

\author{
A thesis submitted in partial fulfillment of the \\ requirements for the degree of \\ MASTERS OF SCIENCE \\ in \\ PHYSICS \\ by
}

Guillermo Matranca

2012 
To: Dean Kenneth G. Furton

College of Arts and Sciences

This thesis, written by Guillermo Matranca, and entitled New Jellium Model for Alkali Metals and its Future Applications to Metal Clusters, having been approved in respect to style and intellectual content, is referred to you for judgment.

We have read this thesis and recommend that it be approved.

Richard Bone

$-\bar{H}$ Hudolf Fiebig

Xuewen Wang, Major Professor

Date of Defense: June 14, 2012

The thesis of Guillermo Matranca is approved.

$\begin{array}{r}\text { Dean Kenneth G. Furton } \\ \text { College of Arts and Sciences }\end{array}$
$\begin{array}{r}\text { Dean Lakshmi N. Reddi } \\ \text { University Graduate School }\end{array}$

Florida International University, 2012 


\section{ACKNOWLEDGMENTS}

I would like to thank the members of my committee for their support on the manuscript and everyone that was around me during this time. They were a major influence to me and it has changed me for the better. This reminds me of a memorable quote once said by a great scientist. If I have seen further it is by standing on the shoulders of giants. I especially like to thank my advisor Dr. Xuewen Wang for being a great mentor and allowing me to expand my horizon. 


\begin{abstract}
OF THE THESIS
NEW JELLIUM MODEL FOR ALKALI METALS AND ITS FUTURE APPLICATIONS TO METAL CLUSTERS

by

Guillermo Matranca

Florida International University

Miami, Florida

Professor Xuewen Wang, Major Professor

This research develops a new method for understanding the properties of
\end{abstract} materials. The new method was applied to alkali metals to examine how well it can predict the Wigner-Seitz radius, rs. Pseudo-potentials for the individual atoms were generated and utilized to obtain the interaction energy within these metals.

The system involves 4 coulombic charges; two of them are the result of the neutral atom (one valence electron and one positive core charge for alkali atoms) and the other two are background charges of equal and opposite amount. This coulombic interaction will behave differently depending on the element that composes the system. There are four groups of energy for this system. One of them has the appearance of the Jellium model, which is solved with Density Functional Theory. From the other three groups, one of them will alter the minimum of the Jellium model for different elements in the system. This group is partially calculated with the help of Ewald summation. This calculation exemplifies that bcc is favored since it is lower in energy than fcc, which is in agreement with experiments for alkali metals. The correction to this energy will be due 
to the core electrons' interaction with a uniform negative charge background. This new method will also be beneficial to calculate the ground state energy of clusters by introducing surface boundaries in the system. 


\section{TABLE OF CONTENTS}

Chapter $1 \quad$ Introduction...............................................................

Experimental and Theoretical

Techniques for Nanoclusters....................................3

Layout of Thesis.......................................................

Chapter 2 Methods for Many-Electron Systems.................................. 8

The Hartree Approximation............................................... 9

The Hartree-Fock Approximation.....................................11

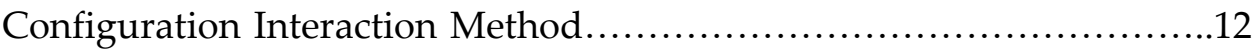

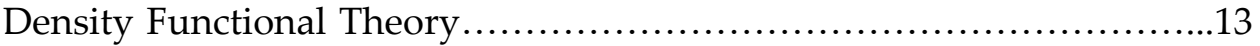

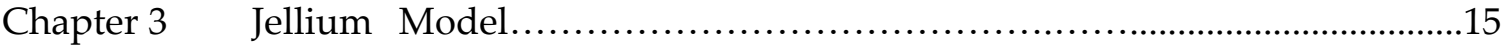

Jellium Model for an Infinite Volume.................................15

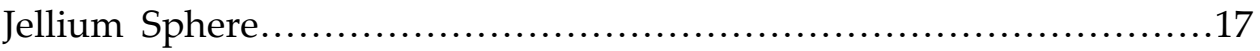

Chapter $4 \quad$ First Principle Pseudo-potential.....................................22

One-dimensional Pseudo-potential....................................23

Pseudo-potential for alkali atoms.....................................26

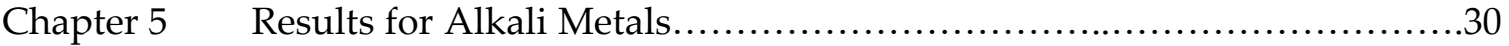

Chapter $6 \quad$ Conclusion......................................................

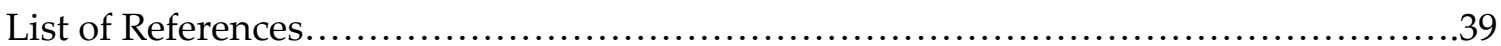




\section{LIST OF TABLES}

TABLE

PAGE

3.1 Physical properties for elements with one valence electron........................17

4.1 Pseudo-potential test for Sodium atom...........................................29

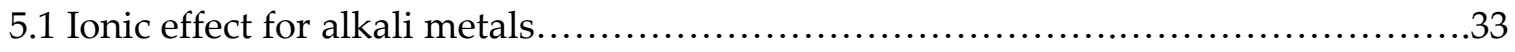

5.2 Minimum $\mathrm{r}_{\mathrm{s}} / \mathrm{a}$ o for alkali metals............................................. 34

5.3 Bulk modulus for alkali metals...............................................35 


\section{LIST OF FIGURES}

FIGURE

PAGE

3.1 Energy versus $\mathrm{r}_{\mathrm{s}} / \mathrm{a}$ o for the Jellium model....................................17

3.2 Flow chart to solve Jellium sphere..............................................19

3.3 Energy versus the number of electrons to the one-third power.....................20

4.1 A pseudo-potential to approximate a coulomb potential..........................22

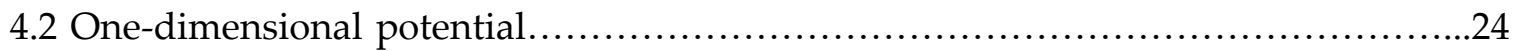

4.3 Energy versus distance for even and odd solutions..............................26

4.4 The selection of the cutoff region in the wavefunction............................. 28

4.5 Total energy of trial 10 against the valence energy

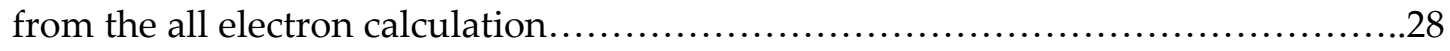

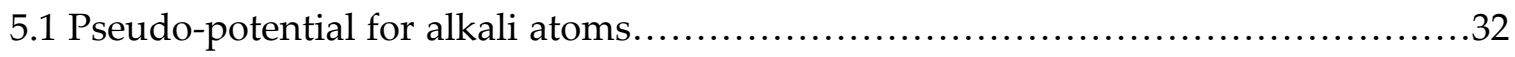

5.2 Total energy versus $\mathrm{r}_{\mathrm{s}} / \mathrm{a}$ o for alkali metals.................................... 


\section{Chapter 1 Introduction}

Properties for bulk materials are so well known and used in many mechanical and electronic instruments today that it gives little opportunity to conduct research on this scale for technological advancements. In order to improve our technology, one can transition the size of material from bulk to small clusters to explore new properties and have a deeper understanding of the material. However, this is a very different realm from bulk, so new conditions must be met when the size is reduced and quantum effects must be considered when finding the characteristic features of these clusters. Currently, scientists are involved in many experiments in parallel with theories for nano-material, but complications still arise because of multiple factors, one being that calculations become too lengthy because the configuration of the system increases exponentially as the number of atoms in the cluster increases. Difficulties tend to also be encountered when models are being used in a long-range spectrum for a system. So, most models that are built usually fit well with experiments in a narrow range of independent parameters. Scientists always try to minimize the computation depending on the system and try to find what variables truly make up the properties of the material being analyzed. This is how theories predict the outcome of experiments; but without experimentation, it is practically impossible to create a theory for phenomena that occur in nature. There still remains an enormous amount of research in the field of nanomaterial, which can also help us understand nature better. 
Nano-material has caught a lot of attention in different fields of science and engineering as a result of the drastic differences in the properties of materials on this scale. This mesoscopic region, which ranges from $1 \mathrm{~nm}$ to $100 \mathrm{~nm}$, is the domain for these nano-size objects and there are many challenges to having a complete understanding of them, and the ability to manipulate them. One major criterion affecting these different properties is the structure and the large surface area-to-volume ratio nano-sized objects contain. This large surface area-to-volume ratio will give different spatial distributions for the electrons and cause the objects to become more reactive than bulk material. For example, noble metals such as $\mathrm{Au}$ and $\mathrm{Ag}$, are great catalysts for chemical reactions when they become ultra fine particles and allow the growth of carbon nanotubes. Another reason for these different properties is the characteristic size of these structures being comparable to the wavelength of the electron. As a result, the system will exhibit quantum confinement. This quantum confinement will make the electrons adjust their energy discretely rather than a continuous manner as in the case of bulk material. Adjusting the size of these structures will show that these discrete energies will have size dependence and stability for a certain number of atoms or molecules in the structure. The numbers of constituents for these stable states are called magic numbers [1]. There are other factors that must be considered in order to understand properties of nano-materials. One is the effect of temperature change and the other is the morphology transitions at a certain time scale 
[2]. However, since my research will involve the study of nano-materials at absolute zero temperature, these two factors are beyond the scope of my thesis.

For this thesis, I will develop a new method to study the lowest energy state (the ground state) for alkali metals in bulk form and compare it with experimental values. My method will also be applicable to alkali metal clusters. I will be able to extend my method to make it applicable to other elements in future research. The major goal is to achieve an understanding of all nano-cluster material, and especially the more exotic ones, which are of interest to scientists because their properties can be used to improve technology.

Quantum Mechanics plays a crucial role in my research and I will present a method of obtaining a solution to the Schrödinger equation since it is not exactly solvable for a many-body system [3]. This approach will not only allow me to analyze nanoclusters but also minimize the computation to get results in a shorter time period. This will benefit the scientific community since efficiency is an important factor in research.

Experimental and Theoretical Techniques for Nanoclusters

First, I will discuss earlier studies at FIU which led to the current project. Then I will give an overview of other approaches for investigating nanoclusters and show the importance of this field.

One such study was to fabricate nano-size cobalt clusters uniformly on a titanium dioxide substrate, a task that proved to be challenging. There were too many 
interactions between the cluster and the substrate that came into play, and these accounted for the lack of uniformity that was produced but also exhibited magic numbers. These magic numbers were encountered through the various experimental techniques that were performed. Another study showed the growth of carbon nanotubes using gold clusters as catalyst. These clusters ranged from 1 to 100 in the nanometer scale and the mechanism of catalytic growth still remains to be completely understood. From these experiments, it was realized that in order to understand the properties of the clusters, one must consider the structure and electronic configuration of these clusters. However, the study of these transition elements is very complex because of the significant d-shell character [4]. In this situation, magnetism needs to be considered. So, one will first have to use simpler systems such as alkali metals as the starting point. Then with this foundation, complex systems can be analyzed by including their additional intrinsic properties.

Techniques to detect nanoclusters still pose many challenges but have improved in recent times. The most common instruments used today are the scanning tunneling microscope (STM), low energy electron diffraction (LEED), atomic force microscopy (AFM), and transmission electron microscopy (TEM). Instruments like LEED follow the principles of diffraction in order to determine the structure of clusters. The interpretations of these diffraction patterns are based on calculations from theoretical constructions of clusters; however, understanding of the patterns is not completely straightforward [5]. Therefore other instruments are necessary to analyze clusters, and 
this will allow scientists to narrow down all the possibilities for the construction of these cluster materials.

There are many approaches to analyze a nano-scale system, but in general, finding the minimum binding energy between atoms within the cluster as the number of atoms increases, can determine the structure at different sizes of the cluster. This can be written in the form

$$
\mathrm{E}_{\mathrm{b}}=\mathrm{a} \mathrm{N}+\mathrm{b} \mathrm{N}^{2 / 3}+\mathrm{c} \mathrm{N}^{1 / 3}+\mathrm{d}
$$

where the first term corresponds to a volume contribution and the other terms represent surface contributions. This equation shows that the major contribution to the binding energy is the volume and surface energy. To find a stable structure, the surface energy must be optimized for a given fixed volume. In a later chapter, I will illustrate how spherical shells give energy stability for a system. For fairly large clusters, Wulff construction can be used to find the optimal structure for stability. Other methods, like the Mackay icosahedron, can optimize the surface by using quasi-spherical shapes or the Marks decahedron approach [6,7]. From experiments, it has been found that the icosahedron shape is more favorable for small clusters and decahedra for intermediate clusters [8]. This could be the reason for the construction of bulk quasi-crystal materials, since these shapes can contain a fivefold axis of symmetry. However, the most favorable structure also depends on the material. Other factors like interaction range, bond order, and bond length must be considered, which can make the icosahedral shape less stable. Also, directionality of the bonds is imortant [9]. 
It has been observed that when the valence electrons are delocalized, as in the alkali and noble metals, electronic shell closing works well. Experiments show this by observing the magic numbers of the system [10]. These clusters are usually called superatoms since they can mimic regular atoms because of the electronic shell configuration. One way to fill these electronic shells is through the spherical jellium model. The spherical jellium model assumes a uniform background of constant positive charge density with which the valence electrons interact, and are constrained because of a spherical boundary. However, filling up these shells fails when clusters are larger than 2000 atoms and geometric shell effects are favored [11,4]. This spherical jellium clearly shows that one model cannot describe a large range of cluster sizes. So, more investigations within this realm must be considered in order to have a better understanding of clusters since the appropriate choice of an energetic model is important.

Other methods to calculate the total energy of nanoclusters have been developed and applied extensively throughout the years. However, these methods can be computationally exhausting. For instance, the Hartree-Fock method can be used to calculate from 2 to 10 electrons in a system but gets computationally cumbersome for larger systems [12]. The density-functional theory (DFT) shows improvement in calculating up to a few hundred atoms in a system. Density-functional theory can even provide the total energy for difficult systems like transition metals and noble metals but it fails if the system is not tested properly since the exchange and correlation term is 
approximated [13]. One approach to this approximation is the local-density approximation (LDA), but an additional term called the gradient correction must be included for complex systems like the transition metals [14].

There are numerous strategies that can be applied to understand systems in the nano-scale but none of them are infallible. This is why there is a vast amount of research being done with material in this area.

\section{Layout of Thesis}

Before discussing my method for analyzing alkali metals, I will explain in Chapter 2 some methods for solving Schrödinger's equation for a many-electron system. In Chapter 3, I will discuss the Jellium model and find the total energy of the system in its ground state for different conditions. This allows me to analyze how bulk material and metal clusters behave. In Chapter 4, I will discuss psuedo-potentials and some applications. Chapter 4 is important for understanding the interaction within the material. What is described in these Chapters is essential for understanding how my method was constructed. In Chapter 5, I will present my results for alkali metals obtained by my method. I will compare these results with experimental results and discuss the similarities and differences. Finally, I will provide discussion on the analysis of clusters with this method. 


\section{Chapter 2 Methods for Many-Electron Systems}

In this chapter, I give an overview of a few important methods that are used to solve Schrödinger's equation for a many electron system. First, I will discuss the oneelectron case. From Quantum Mechanics, the time-independent Schrödinger equation has the form

$$
\frac{-\hbar^{2}}{2 m} \nabla^{2} \psi(\mathbf{r})+U(\mathbf{r}) \psi(\mathbf{r})=\mathrm{E} \psi(\mathbf{r})
$$

where $U(\mathbf{r})$ is the potential energy for the single-electron. This potential is the result of all the other particles in the system and any external potential acting on this electron. Choosing an appropriate $U(\mathbf{r})$ for a real system like metal is a difficult task. A mean field for the potential can be used but this would not describe an accurate picture for the electron. In order to have a more accurate calculation, the wavefunction should involve all N-particles of the system that are non-static. Therefore, for a fully interacting, manyelectron system with fixed ions, the Schrödinger equation is

$$
\sum_{i=1}^{N}\left(\frac{-\hbar^{2}}{2 m} \nabla_{i}^{2} \Psi-\frac{1}{4 \pi \varepsilon_{\circ}} \sum_{k} \frac{Z_{k} e^{2}}{\left|\mathbf{r}_{i}-\mathbf{R}_{k}\right|} \Psi\right)+\frac{1}{2} \sum_{i=1}^{N} \sum_{\substack{j=1 \\ j \neq i}}^{N} \frac{e^{2}}{4 \pi \varepsilon_{\circ}\left|\mathbf{r}_{i}-\mathbf{r}_{j}\right|} \Psi=\mathrm{E}_{\text {total }} \Psi
$$

where $\Psi$ is the N-electron wavefunction, which is a function of the position of each electron. $Z_{k}$ are the ionic charges, $\mathbf{R}_{k}$ are the positions of the ions and $\mathbf{r}_{i}$ are the electrons' positions. The second and last term on the left-hand side of the equation represents the attractive electrostatic potential and the repulsive potential between electrons, respectively. However, this equation cannot be solved exactly. 
Approximations must be considered and the solution obtained from these approximation methods should provide an accurate picture for the particular problem. The single-electron equation is usually a good starting point when the potential is not complicated. For instance, when the particles are non-interacting, the Schrödinger equation can be solved exactly. However, real systems tend to be intricate because of the repulsive interaction between the electrons.

\section{The Hartree Approximation}

For this approach, the electrons in the system are considered to be independent of each other so that the electronic charge density will simply be the sum of each of their modulus squared stationary states associated with their charge. The form is shown to be,

$$
\rho(\mathbf{r})=\sum_{j=1}^{N}(-e)\left|\psi_{j}(\mathbf{r})\right|^{2}
$$

This type of charge density is used for the electronic potential of the system. If the $i^{\text {th }}$ particle is removed from the charge density, then the potential energy will simply be the electric potential that results from all but the $i^{\text {th }}$ electron multiplied by the charge of the $i^{\text {th }}$ electron:

$$
\begin{gathered}
U^{e l}(\mathbf{r})=(-e) \cdot \phi_{i}(\mathbf{r}) \\
\text { where, } \phi_{i}(\mathbf{r})=\int \frac{\rho\left(\mathbf{r}^{\prime}\right)}{\left|\mathbf{r}_{i}-\mathbf{r}^{\prime}\right|} d \mathbf{r}^{\prime} \text {, and } \rho(\mathbf{r})=\sum_{\substack{j=1 \\
j \neq i}}^{N}(-e)\left|\psi_{j}\left(\mathbf{r}^{\prime}\right)\right|^{2}
\end{gathered}
$$

Here, the electric potential of the remaining electrons is treated as a smooth distribution of negative charge since the stationary states are considered continuous with position. 
The Hartree approximation also includes the interaction energy between the ionic charge and electron charge,

$$
U_{i o n}\left(\mathbf{r}_{i}\right)=\frac{-1}{4 \pi \varepsilon_{\circ}} \sum_{k} \frac{Z_{k} e^{2}}{\left|\mathbf{r}_{i}-\mathbf{R}_{k}\right|}
$$

The Hartree equation incorporates these two potential energies to the potential of a single-electron equation. For $N$-electrons there will be $N$ equations:

$$
\begin{aligned}
& \frac{-\hbar^{2}}{2 m} \nabla_{i}^{2} \psi_{i}\left(\mathbf{r}_{i}\right)+U_{i o n}\left(\mathbf{r}_{i}\right) \psi_{i}\left(\mathbf{r}_{i}\right)+\left[\int \sum_{\substack{j=1 \\
j \neq i}}^{N} \frac{e^{2}\left|\psi_{j}\left(\mathbf{r}^{\prime}\right)\right|^{2}}{\left|\mathbf{r}_{i}-\mathbf{r}^{\prime}\right|} d \mathbf{r}^{\prime}\right] \psi_{i}\left(\mathbf{r}_{i}\right)=\mathrm{E}_{i} \psi_{i}\left(\mathbf{r}_{i}\right) \\
& i=1,2,3, \ldots \ldots, N .
\end{aligned}
$$

The potential for each equation is different resulting in the single-electron wavefunctions not being orthogonal to each other. In order to satisfy orthogonality conditions, the charge density must be approximated with an average of the orbital density, which will lead to having only one equation. Now the Hartree equation is shown to be

$$
\frac{-\hbar^{2}}{2 m} \nabla_{i}^{2} \psi_{i}\left(\mathbf{r}_{i}\right)+U_{i o n}\left(\mathbf{r}_{i}\right) \psi_{i}\left(\mathbf{r}_{i}\right)+\left[\int \frac{N-1}{N} \sum_{j=1}^{N} \frac{e^{2}\left|\psi_{j}\left(\mathbf{r}^{\prime}\right)\right|^{2}}{\left|\mathbf{r}_{i}-\mathbf{r}^{\prime}\right|} d \mathbf{r}^{\prime}\right] \psi_{i}\left(\mathbf{r}_{i}\right)=\mathrm{E}_{i} \psi_{i}\left(\mathbf{r}_{i}\right)
$$

This equation clearly shows non-linearity. To get a solution from this equation, a selfconsistent approach is followed by finding a form for the electronic density and using it to get a solution for the one-electron wavefunction [15]. The wavefunction is then put back into the electronic density to get a potential, where the potential is used to solve Hartree equation again. This iteration is continued until the potential is consistent. This 
method can give accurate results but numerical complications arise even though this equation is a crude approximation of the full Schrödinger equation. The physical feature cannot be fully described since this method assumes independent electrons. Therefore other properties must be included in order to obtain a better picture of the system.

\section{The Hartree-Fock Approximation}

One condition the Hartree equation does not consider for the electrons is the antisymmetrization of the electrons because of the Pauli principle for fermions. The wavefunction in the Hartree equation has the form

$$
\Psi\left(\mathbf{r}_{1} s_{1}, \mathbf{r}_{2} s_{2}, \ldots \ldots, \mathbf{r}_{N} s_{N}\right)=\psi_{1}\left(\mathbf{r}_{1} s_{1}\right) \cdot \psi_{2}\left(\mathbf{r}_{2} s_{2}\right) \cdots \cdots \psi_{N}\left(\mathbf{r}_{N} s_{N}\right)
$$

for an $\mathrm{N}$ particle system. The wavefunction needs to follow the condition where

$$
\Psi\left(\mathbf{r}_{1} s_{1}, \ldots, \mathbf{r}_{i} s_{i}, \ldots, \mathbf{r}_{j} s_{j}, \ldots, \mathbf{r}_{N} s_{N}\right)=-\Psi\left(\mathbf{r}_{1} s_{1}, \ldots, \mathbf{r}_{j} s_{j}, \ldots, \mathbf{r}_{i} s_{i}, \ldots, \mathbf{r}_{N} s_{N}\right)
$$

Applying Slater's Determinant for the stationary orbitals can satisfy this condition.

$$
\Psi\left(\mathbf{r}_{1} s_{1}, \mathbf{r}_{2} s_{2}, \ldots \ldots, \mathbf{r}_{N} s_{N}\right)=\frac{1}{\sqrt{N !}}\left|\begin{array}{cccc}
\psi_{1}\left(\mathbf{r}_{1} s_{1}\right) & \psi_{1}\left(\mathbf{r}_{2} s_{2}\right) & \cdots \cdots & \psi_{1}\left(\mathbf{r}_{N} s_{N}\right) \\
\psi_{2}\left(\mathbf{r}_{1} s_{1}\right) & \psi_{2}\left(\mathbf{r}_{2} s_{2}\right) & \cdots \cdots & \psi_{2}\left(\mathbf{r}_{N} s_{N}\right) \\
\vdots & & & \vdots \\
\psi_{N}\left(\mathbf{r}_{1} s_{1}\right) & \psi_{N}\left(\mathbf{r}_{2} s_{2}\right) & \cdots \cdots & \psi_{N}\left(\mathbf{r}_{N} s_{N}\right)
\end{array}\right|
$$

The Hartree-Fock approximation applies equation (2.10) to the full electron wavefunction and minimizes the expectation value of the Hamiltonian with respect to the single-electron wavefunction:

$$
\delta\left[\langle\Psi|\hat{\mathbf{H}}| \Psi\rangle-\sum_{i} \mathrm{E}_{i} \int\left|\psi_{i}\right|^{2} d \mathbf{r}^{\prime}\right]=0
$$

The minimization leads to the Hartree-Fock equations, 


$$
\begin{aligned}
& \left(\frac{-\hbar^{2}}{2 m} \nabla^{2}+U_{i o n}(\mathbf{r})\right) \psi_{i}(\mathbf{r})+\left[\int \sum_{j=1}^{N} \frac{e^{2}\left|\psi_{j}\left(\mathbf{r}^{\prime}\right)\right|^{2}}{\left|\mathbf{r}_{i}-\mathbf{r}^{\prime}\right|} d \mathbf{r}^{\prime}\right] \psi_{i}(\mathbf{r}) \\
& -\sum_{j} \delta_{s_{i} s_{j}} \int \frac{e^{2} \psi_{j}^{*}\left(\mathbf{r}^{\prime}\right) \cdot \psi_{i}\left(\mathbf{r}^{\prime}\right)}{\left|\mathbf{r}-\mathbf{r}^{\prime}\right|} d \mathbf{r}^{\prime} \psi_{j}(\mathbf{r})=\mathrm{E}_{i} \psi_{i}(\mathbf{r}) .
\end{aligned}
$$

Equation (2.12) is similar to the Hartree equation, which only differs by the last term on the left-hand side of the equation. The second and third terms are known as the direct and exchange term, respectively. Note also that the self-interacting term cancels because of the exchange term. The exchange term only contributes when the spin state of the $j^{\text {th }}$ electron is parallel to the spin state of the $i^{\text {th }}$ electron. The Hartree-Fock equations are difficult to solve because the exchange term is a non-local integral operator and only a few cases are manageable. For instance, choosing a set of orbitals to be orthonormal plane waves for a constant periodic potential can solve the Hartree-Fock exactly.

\section{Configuration Interaction Method}

There are many extensions to Hartree-Fock approach but the one most often used is the Configuration Interaction (CI). This method applies a linear combination of N-electron Slater determinants to the wavefunction [16]. The first term is simply the Hartree-Fock Slater determinant, while the following terms are excited states of the virtual Hartree-Fock orbitals. However, this approach tends to scale very poorly when the system size increases and is related to the binomial coefficient:

$$
\left(\begin{array}{l}
M \\
N
\end{array}\right)=\frac{M !}{N !(M-N) !}
$$


where $\mathrm{M}$ is the number of Hartree-Fock orbitals and $\mathrm{N}$ is the number of electrons. This method can be highly accurate for small systems but, as $\mathrm{N}$ increases, CI calculations require a lot of computational power.

\section{Density Functional Theory (DFT)}

The Density Functional Theory, which is formally an exact theory, is based on the charge density of a system. It also states that the charge density of a system cannot have two or more distinct potentials [17]. The potential is unique for the particular charge density. For such a system, the particle density for the ground state is given by

$$
\rho_{G}(\mathbf{r})=N \int\left|\Psi_{G}\left(\mathbf{r}, \mathbf{r}_{2}, \ldots, \mathbf{r}_{N}\right)\right|^{2} d \mathbf{r}_{2} \ldots d \mathbf{r}_{N}
$$

Now, for the single-electron equation, the potential is written differently.

$$
\begin{aligned}
& {\left[\frac{-\hbar^{2}}{2 m} \nabla^{2}+\mathrm{V}(\mathbf{r})\right] \psi_{i}(\mathbf{r})=\mathrm{E}_{i} \psi_{i}(\mathbf{r}), \text { where, }} \\
& \mathrm{V}(\mathbf{r})=\mathrm{V}_{e x t}(\mathbf{r})+\mathrm{V}_{H}(\mathbf{r})+\mathrm{V}_{X C}[\rho(\mathbf{r})] .
\end{aligned}
$$

$\mathrm{V}_{e x t}(\mathbf{r})$ is the external potential associated with the ions, $\mathrm{V}_{H}(\mathbf{r})$ is the direct potential term given by the Hartree equation, and $\mathrm{V}_{X C}[\rho(\mathbf{r})]$ is the exchange and correlation potential. Equation (2.15) will allow expressing the energy of the system as a functional.

$$
\begin{aligned}
\mathrm{E}[\rho(\mathbf{r})]= & \frac{-\hbar^{2}}{2 m} \sum_{i=1}^{N} \int \psi_{i}^{*}(\mathbf{r}) \nabla^{2} \psi_{i}(\mathbf{r}) d \mathbf{r}+\mathrm{E}_{X C}[\rho(\mathbf{r})]+\int \rho(\mathbf{r}) \mathrm{V}_{e x t}(\mathbf{r}) d \mathbf{r} \\
& +\frac{e^{2}}{2} \iint \frac{\rho(\mathbf{r}) \rho\left(\mathbf{r}^{\prime}\right)}{\left|\mathbf{r}-\mathbf{r}^{\prime}\right|} d \mathbf{r} d \mathbf{r}^{\prime}
\end{aligned}
$$


However, DFT assumes that the exchange-correlation functional is known but it has only been determined numerically for a few simple model systems. Therefore, most of the density functional calculations use the Local Density Approximation (LDA). The LDA approximates the exchange-correlation functional with a uniform homogeneous electron gas of density $n(\mathbf{r})$ at any position $\mathbf{r}$. The expression for $\mathrm{E}_{X C}[n(\mathbf{r})]$ using LDA is

$$
\mathrm{E}_{X C}[n(\mathbf{r})] \approx \int \varepsilon_{X C}(n(\mathbf{r})) \cdot n(\mathbf{r}) d \mathbf{r} .
$$

This approximation can give very accurate values for the system but often fails when the system has electrons that are strongly correlated, as in states of electrons containing $d$ and $f$ orbitals. 


\section{Chapter 3 Jellium Model}

When solving a many-electron problem that contains many ions in the system, a

large degree of freedom in terms of crystalline structure arises. This large degree of freedom makes the Schrödinger equation difficult to solve. In this chapter, I will discuss the Jellium model, which is an approximation to the ions, as a means of simplifying the system. This model will be solved for a volume of infinite size and for a spherical boundary.

Jellium Model for an Infinite Volume

The Jellium model, which is a much cruder model to describe metals, can also be used to find properties for a system, but only when the system is large or comparable to bulk. The theory behind this model replaces the structural ions, which have localized charge, with a uniform charge distribution throughout the region of the material. This so-called "background" charge distribution will interact with the electrons of the material [18]. The Hamiltonian for this model with $N$-electrons confined in space of volume $V$ is as follows.

$$
\begin{gathered}
\hat{H}=\hat{H}_{e l}+\hat{H}_{b a c k}+\hat{H}_{e l-b a c k}, \text { where } \\
\hat{H}_{e l}=\sum_{i=1}^{N} \frac{\hat{p}_{i}^{2}}{2 m}+\sum_{\substack{i=1 \\
i \neq j}}^{N} \frac{e^{2}}{\left|\mathbf{r}_{i}-\mathbf{r}_{j}\right|}, \\
\hat{H}_{b a c k}=\frac{e^{2}}{2} \int_{V} \int_{V} \frac{n(\mathbf{R}) \cdot n\left(\mathbf{R}^{\prime}\right)}{\left|\mathbf{R}-\mathbf{R}^{\prime}\right|} d \mathbf{R}^{\prime} d \mathbf{R}=\frac{e^{2}}{2}\left(\frac{N}{V}\right)^{2} \int_{V} d \mathbf{R} \int_{V} d \mathbf{R}^{\prime}, \\
\hat{H}_{e l-b a c k}=\int_{V} \int_{V} \frac{\rho(\mathbf{r}) \cdot n(\mathbf{R})}{|\mathbf{r}-\mathbf{R}|} d \mathbf{R} d \mathbf{r}=-e^{2} \frac{N}{V} \sum_{i=1}^{N} \int_{V} \frac{1}{\left|\mathbf{r}_{\mathrm{i}}-\mathbf{R}\right|} d \mathbf{R} .
\end{gathered}
$$


$\rho(\mathbf{r})$ is the electronic density and $n(\mathbf{R})$ is the background charge density. As a result of the uniformity of the charges and a volume of infinite size, this will allow the wavefunction to be a set of free electron plane waves. The solution for the energy of the system using the Hartree-Fock approximation will have a form of

$$
\frac{\mathrm{E}}{N}=\left[\frac{3}{5} \frac{(9 \pi / 4)^{2 / 3}}{\left(\mathrm{r}_{\mathrm{s}} / \mathrm{a}_{\circ}\right)^{2}}-\frac{3}{2 \pi} \frac{(9 \pi / 4)^{1 / 3}}{\left(\mathrm{r}_{\mathrm{s}} / \mathrm{a}_{\circ}\right)}\right] \mathrm{Ry} .
$$

$\mathrm{a}_{\circ}$ is the Bohr radius and $r_{s}$ is the Wigner Seitz radius. The Wigner Seitz radius is related to the average uniform density of the system given as $\frac{1}{N / V}=\frac{4 \pi}{3} \mathrm{r}_{\mathrm{s}}{ }^{3}$. The units for energy are in rydberg (Ry), where $1 \mathrm{Ry}=13.6 \mathrm{eV}$. To correct the Hartree-Fock result, exact leading terms in a high-density expansion are added.

$$
\frac{\mathrm{E}}{N}=\left[\frac{3}{5} \frac{(9 \pi / 4)^{2 / 3}}{\left(\mathrm{r}_{\mathrm{s}} / \mathrm{a}_{\circ}\right)^{2}}-\frac{3}{2 \pi} \frac{(9 \pi / 4)^{1 / 3}}{\left(\mathrm{r}_{\mathrm{s}} / \mathrm{a}_{\circ}\right)}+0.0622 \ln \left(\mathrm{r}_{\mathrm{s}} / \mathrm{a}_{\circ}\right)-0.096+O\left(\mathrm{r}_{\mathrm{s}} / \mathrm{a}_{\circ}\right)\right] \mathrm{Ry}
$$

These corrections required much labor [19]. The last three terms are called the correlation energy, which is a misnomer since they have no physical meaning. Figure 3.1 shows the energy per atom as a function of $r_{s} / a_{\circ}$ using the Jellium model for a volume of infinite size. The minimum in energy occurs at a Wigner Seitz radius of $3.83 \mathrm{a}_{\circ}$, which is constant in this model for any atom that composes the system. The Wigner Seitz radius does not agree with the experimental values as shown in Table 3.1. Table 3.1 only lists a few elements from the periodic table that have one electron in the conduction band, and shows other properties for these elements that will be used for analysis later 


\begin{tabular}{ccccc}
\hline Elements & $\begin{array}{c}\text { Lattice } \\
\text { constant } \\
\mathrm{a}(\AA)\end{array}$ & $\begin{array}{c}\text { Wigner } \\
\text { Seitz } \\
\text { radius } \\
\mathrm{r}_{\mathrm{s}}(\AA)\end{array}$ & $\begin{array}{c}\text { Bulk } \\
\text { modulus B } \\
\left(10^{11} \mathrm{~N} / \mathrm{m}^{2}\right)\end{array}$ & $\begin{array}{c}\text { Crystal } \\
\text { structure }\end{array}$ \\
\hline${ }^{*} \mathrm{H}$ & 3.27 & 1.61 & & $\mathrm{bcc}$ \\
$\mathrm{Li}$ & 3.49 & 1.72 & 0.116 & $\mathrm{bcc}$ \\
$\mathrm{Na}$ & 4.23 & 2.08 & 0.068 & $\mathrm{bcc}$ \\
$\mathrm{K}$ & 5.23 & 2.57 & 0.032 & $\mathrm{bcc}$ \\
$\mathrm{Rb}$ & 5.59 & 2.75 & 0.031 & $\mathrm{bcc}$ \\
$\mathrm{Cs}$ & 6.05 & 2.98 & 0.020 & $\mathrm{bcc}$ \\
$\mathrm{Cu}$ & 3.61 & 1.41 & 1.37 & $\mathrm{fcc}$ \\
$\mathrm{Ag}$ & 4.09 & 1.60 & 1.007 & $\mathrm{fcc}$ \\
$\mathrm{Au}$ & 4.08 & 1.59 & 1.732 & $\mathrm{fcc}$ \\
\hline
\end{tabular}

Table 3.1. * The values obtained for metal Hydrogen are calculated theoretically.

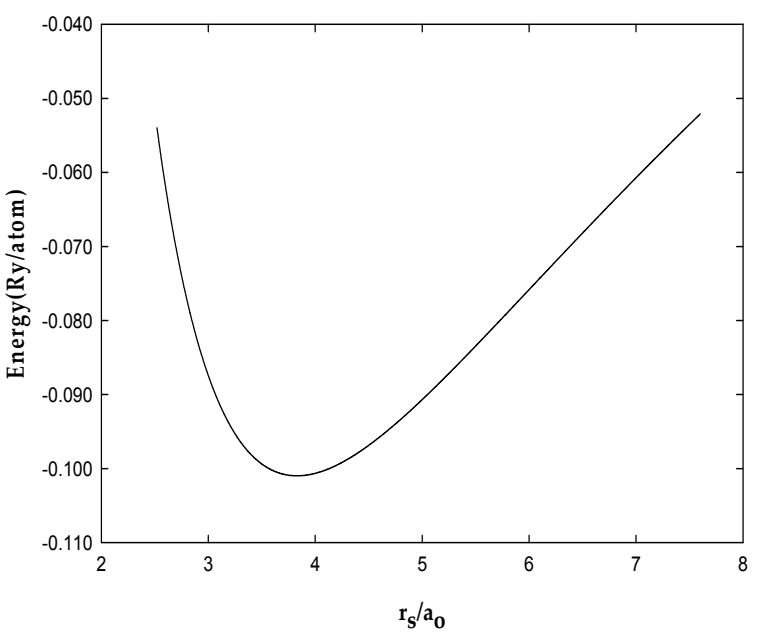

Figure 3.1. The plot shows the minimum energy per atom for a uniform charge density occurs at 3.83 .

in my research. The reason for this disagreement is that real systems lack homogeneity whereas Jellium assumes uniformity for the ionic structure. In order to incorporate this effect, one can consider replacing this uniform background with some form of potential that can describe the ionic structure of the system, but then this will increase the complication of computational calculation where the wavefunction will not have the form of a plane wave.

\section{Jellium Sphere}

In the previous section, I discussed the Jellium model involving the material occupying all of space. This condition and applying the Hartree-Fock approximation allowed the Jellium to be solved. Then the correlation terms were included to have a more accurate calculation for the electron-electron interaction. The total energy per atom for this system depends only on the density. In contrast, I will consider a boundary, 
constrained to a sphere, for the Jellium model. This will input an additional parameter, which I will use to investigate the energy of the system. This variable parameter will pertain to the radius of the sphere, which is proportional to the number of atoms to the one-third power in the system. However, having this constraint, the Schrödinger equation needs to be solved numerically by a self-consistent approach. A trial function along with an assumed potential associated with the charges will initiate the iteration. For this system, the total potential energy is given as

$$
\mathrm{U}(\mathbf{r})=\mathrm{U}_{\mathrm{ext}}(\mathbf{r})+\mathrm{U}_{\mathrm{H}}(\mathbf{r})+\mathrm{U}_{\mathrm{XC}-\mathrm{LDA}}[\rho(\mathbf{r})] .
$$

$\mathrm{U}_{\text {ext }}(\mathbf{r})$ is the potential energy of the positive charges distributed uniformly within the sphere that interacts with the electrons. $\mathrm{UH}_{\mathrm{H}}(\mathbf{r})$ is the Hartree term, also called the direct term, and is expressed as

$$
\mathrm{U}_{\mathrm{H}}(\mathbf{r})=\int \frac{N-1}{N} \sum_{j=1}^{N} \frac{e^{2}\left|\psi_{j}\left(\mathbf{r}^{\prime}\right)\right|^{2}}{\left|\mathbf{r}_{i}-\mathbf{r}^{\prime}\right|} d \mathbf{r}^{\prime}=\frac{N-1}{N}(-e) \int \frac{\rho\left(\mathbf{r}^{\prime}\right)}{\left|\mathbf{r}_{i}-\mathbf{r}^{\prime}\right|} d \mathbf{r}^{\prime}
$$

$\mathrm{UxC-LDA}[\rho(\mathbf{r})]$ is the exchange-correlation potential, which is a functional of density, and is approximated by the LDA. In order to solve the Schrödinger equation, an electronic charge density must be given. Since the density is related to the single-particle wavefunction, $\rho\left(\mathbf{r}^{\prime}\right)=\sum_{j=1}^{N}(-e)\left|\psi_{j}\left(\mathbf{r}^{\prime}\right)\right|^{2}$, a trial wavefunction can be given first. Then the potential energy of the system can be solved using this density. This potential is 


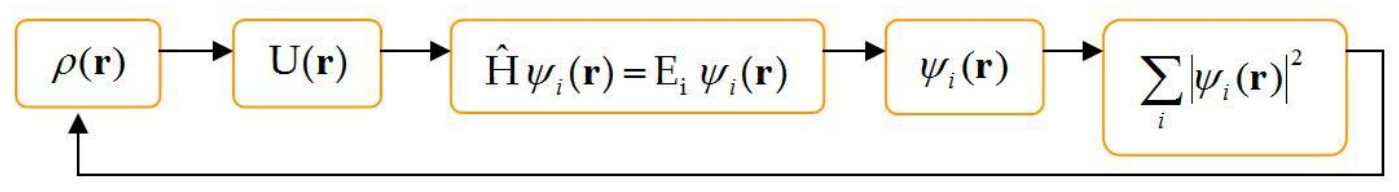

Figure 3.2. Flow chart to solve Jellium sphere.

substituted in Schrödinger's equation to get a solution. The process can continue by using the solution to get the new density. A flow chart is shown in figure 3.2. A program written in FORTRAN code was used to generate a loop for this iteration until the result converged to a stable value. From this, the eigenvalues can be summed to get to total energy of the system. This simulation is executed every time when a new number of charges are added to the system.

Two cases will be considered. For the first case, the positive charge density value will be fixed throughout the sphere and $r_{s}$ will vary smoothly while the average electronic charge density is the same as the positive charge density. The second case will consist of having a spherical shell at the outer end of the sphere with a certain thickness that depends on the inter-planer distance of the atoms. The $r_{s}$ value within the spherical shell will vary while $r_{s}$ of the sphere inscribed in the shell will be fixed. This variable $r_{s}$ will have a limited range depending on the thickness of the shell and the fixed $r_{s}$ value within the sphere. In notation terms, $\left(\frac{3 \mathrm{~V}}{4 \pi}\right)^{1 / 3} \leq \mathrm{r}_{\mathrm{svar} .} \leq \mathrm{r}_{\mathrm{sfix}}$, where $\mathrm{V}$ is the volume of 


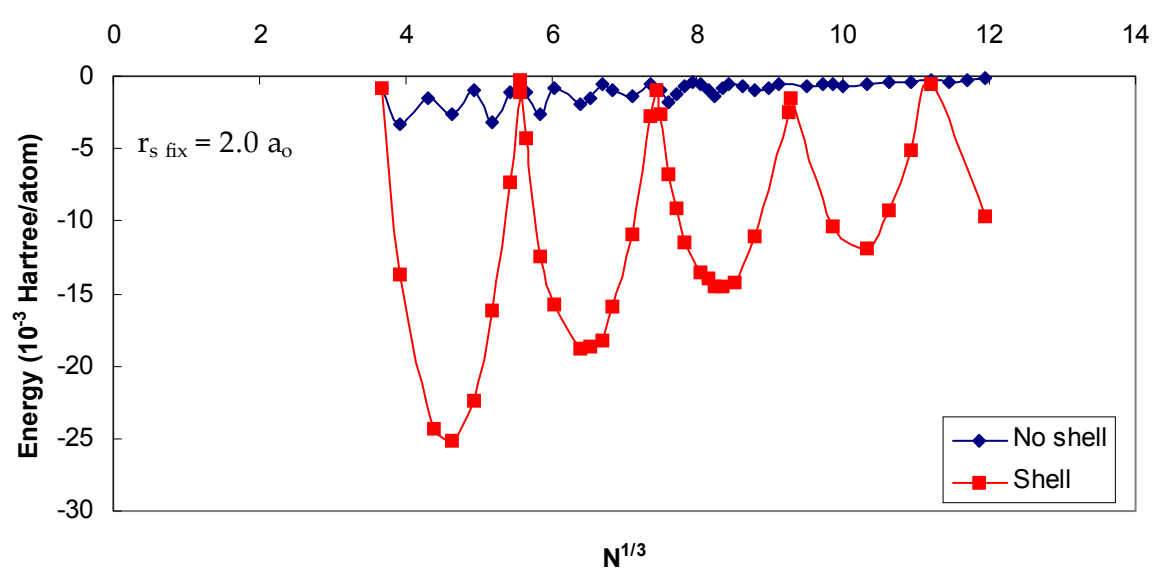

Figure 3.3. Energy versus the number of electrons to the one-third power. The energy units are in Hartree $(1$ Hartree $=$ 2 Ry). The shell model shows larger energy difference, which indicates stability.

the shell. Adding this spherical shell to the model mimics the surface effects that occur in real systems. The data for the two cases are superimposed on one plot to see their differences. The plot is shown in figure 3.3 for $r_{s}$ fix $=2.0 a_{\circ}$. A new shell gets added for the next atom when $\mathrm{r}_{\mathrm{s} \text { var }}$ reaches $2.0 \mathrm{a}_{\circ} . \mathrm{N}$ is the number of atoms in the system and is related to the radius, $R$, of the sphere, by $N \frac{4}{3} \pi r_{s}^{3}=\frac{4}{3} \pi R^{3}$. The shell model shows large oscillations compared to the case without a spherical shell. Having this large energy difference produces stability of a system at certain discrete values of N. These discrete values are called magic numbers. The energy difference needs to be larger than the energy at room temperature, which is about one milli-Hartree, in order for the system to be stable. Without the surface effect, the Jellium sphere clearly shows instability. In addition, this also shows that this "core" region has negligible effects so it can be replaced with a constant. The same effect occurs with the shell model when $\mathrm{N}$ is large, which should be the case since the energy of the infinite jellium is fixed for a given 
$r_{s}$ value. The shell model is a good approximation for understanding the stability of clusters but does not indicate correctly the magic numbers. It also overestimated the energy difference for certain magic numbers. Ignoring the structure of clusters gives a major discrepancy to the model but should not be completely disregarded.

I will continue to use the Jellium model for alkali metals; however, I will not ignore the lattice structure. Including the lattice structure will give different $r_{s}$ minimum values for different elements that compose the bulk system. My scheme will be explained in detail in chapter 5, but first I need to discuss the pseudo-potential, which is also an essential method for my analysis of alkali metals. Pseudo-potential will be applied to the alkali atoms to describe how charges interact in the core region of the atoms. 


\section{Chapter 4 First Principle Pseudo-potential}

When calculations require substantial computational power, as in many-body problems, it is useful to apply the Pseudo-potential method. This method helps reduce calculations by introducing a cut-off to the system where it is least significant. The

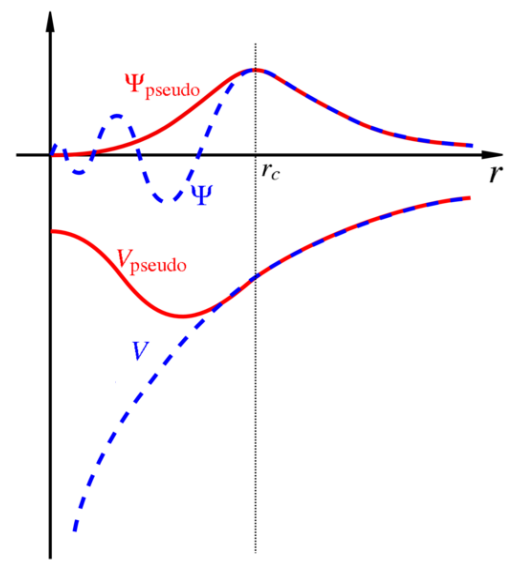

Figure 4.1. A pseudo-potential to approximate a coulomb potential. approximated system has to satisfy certain conditions in

order for calculations to not deviate from the actual results in the region of interest. The features of pseudopotentials are usually used to remove the core electrons in the system. Removal of core electrons allows the valence electrons to be the main contribution since they tend to be the ones that interact the most with outside

sources. Consider a coulombic potential between the electrons and the nuclei as shown in figure 4.1. The pseudo-potential will differ from the region of the coulombic potential where the wavefunction has rapid oscillations, which is considered to be the core electrons interacting with the nuclei. Outside this region of the core, the pseudopotential must fit the true potential exactly. This is where valence electrons are located and the wavefunction is unaltered in this region. In order to have a satisfactory pseudopotential, the integral of the squared amplitude, where the core electrons are located, has to match as in the case of the real potential. This is known as the norm-conservation [20]. Another condition that the pseudo-potential must satisfy is the charge density in the valence region should be identical to the true charge density. 


\section{$\underline{\text { One-dimensional Pseudo-potential }}$}

When generating a pseudo-potential, it is important to determine which parts are essential for your system and this is usually simple when a potential is symmetric. More importantly is the transferability when using the pseudo-potential. I will describe a special case in detail since the pseudo-potential is an important factor for my analysis of alkali metals. I consider a one-dimensional double-well harmonic oscillator interconnected by a constant potential $\mathrm{V}_{\circ}$.

$$
\mathrm{V}=\left\{\begin{array}{cc}
\frac{1}{2}\left(x-x_{\circ}\right)^{2}, & x>x_{4}, \\
\mathrm{~V}_{\circ}, & x_{3}<x<x_{4}, \\
\frac{1}{2}\left(x+x_{\circ}\right)^{2}, & x<x_{3},
\end{array}\right.
$$

where the spring constants are set equal to 1 . Now, for the pseudo-potential I have two harmonic oscillators cut in half with an infinite potential and also interconnected with the same constant potential $\mathrm{V}_{\circ}$.

$$
\mathrm{V}_{\text {pseudo }}=\left\{\begin{array}{cc}
\infty, & |x|>x_{\circ}, \\
\frac{1}{2}\left(x-x_{\circ}\right)^{2}, & x_{4}<x<x_{\circ}, \\
\mathrm{V}_{\circ}, & x_{3}<x<x_{4}, \\
\frac{1}{2}\left(x+x_{\circ}\right)^{2}, & x_{\circ}<x<x_{3} .
\end{array}\right.
$$

These two potentials are shown in figure 4.2. I will call $\mathrm{V}$ and $\mathrm{V}_{\text {pseudo }}$ full harmonic and half harmonic respectively. Accordingly, the full and half harmonic wavefunctions are $\psi_{\text {full }}$ and $\psi_{\text {half }}$ respectively. Using the WKB approximation, their solutions are 


$$
\begin{aligned}
\psi_{\text {full }}= & \frac{1}{\sqrt{\kappa}} \cos \left(\int_{x_{1}}^{x_{2}} \mathrm{k} d x-\frac{\pi}{2}\right) \cdot \exp \left(-\int_{x_{2}}^{x} \kappa d x\right) \\
& -\frac{2}{\sqrt{\kappa}} \sin \left(\int_{x_{1}}^{x_{2}} \mathrm{k} d x-\frac{\pi}{2}\right) \cdot \exp \left(\int_{x_{2}}^{x} \kappa d x\right)
\end{aligned}
$$

$$
\begin{aligned}
\psi_{\text {half }}= & \frac{1}{2 \sqrt{\kappa}} \sin \left(\int_{x_{\circ}}^{x_{2}} \mathrm{k} d x-\frac{\pi}{4}\right) \cdot \exp \left(-\int_{x_{2}}^{x} \kappa d x\right) \\
& +\frac{1}{\sqrt{\kappa}} \cos \left(\int_{x_{\circ}}^{x_{2}} \mathrm{k} d x-\frac{\pi}{4}\right) \cdot \exp \left(\int_{x_{2}}^{x} \kappa d x\right)
\end{aligned}
$$
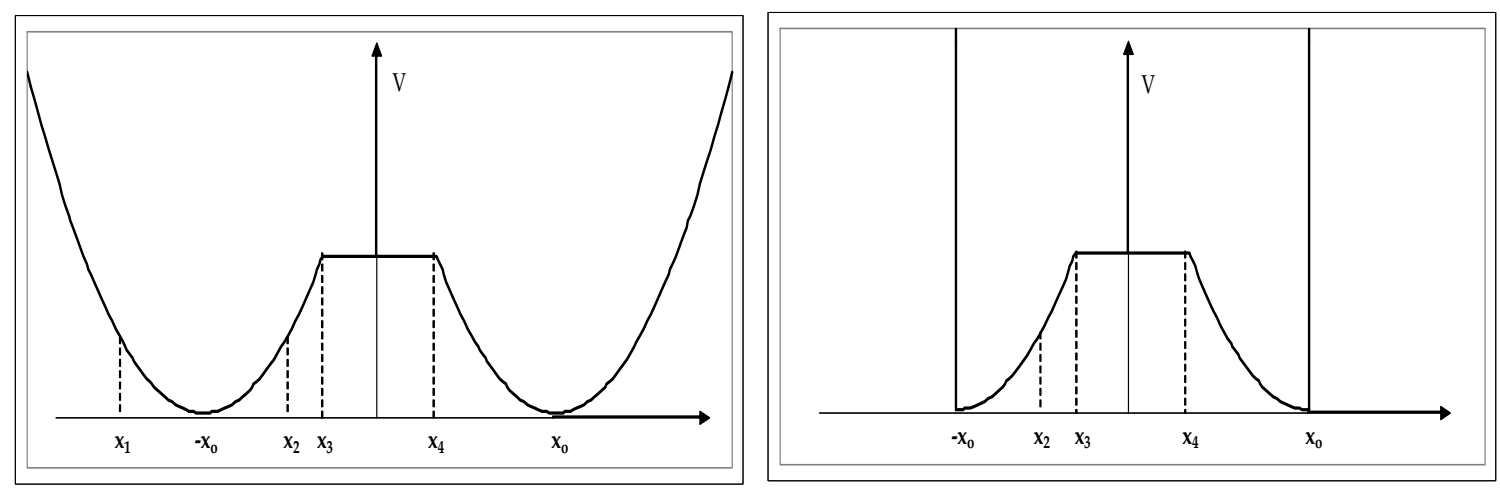

Figure 4.2. Shows a double harmonic potential interconnected with a constant potential and two half harmonic potential also interconnected by constant potential.

where, $\mathrm{k}=\frac{\sqrt{2 m}}{\hbar} \sqrt{\mathrm{E}-\mathrm{V}(x)}$, and $\kappa=\frac{\sqrt{2 m}}{\hbar} \sqrt{\mathrm{V}(x)-\mathrm{E}}$. Now, since the potential is symmetric, the wavefunctions can always be taken to be either even or odd. So, two conditions must be satisfied: $\psi(0)=0$ for odd solutions and $\psi^{\prime}(0)=0$ for even solutions. This will lead to two transcendental equations for full harmonic and also for the half harmonic oscillator. The equations are shown to be, 


$$
\begin{aligned}
& \pm 2 \tan \left(\int_{x_{1}}^{x_{2}} \mathrm{k} d x-\frac{\pi}{2}\right)=\exp \left(-2 \int_{x_{2}}^{0} \kappa d x\right) \quad \text { (full harmonic), } \\
& \mp 2 \cot \left(\int_{x_{0}}^{x_{2}} \mathrm{k} d x-\frac{\pi}{4}\right)=\exp \left(-2 \int_{x_{2}}^{0} \kappa d x\right) \quad \text { (half harmonic). }
\end{aligned}
$$

The plus term for the full harmonic is the result of the odd solution while the minus term for the half harmonic is from the odd solution. Plotting both sides of the equation individually on the same graph to get intersecting points will provide the energy values for the system. The two systems, the full and the half, show degeneracy splitting when the distances between the potential of zero value approach each other. Also, the energy between the even and odd solutions for the different potentials shows a greater difference when the constant potential interconnecting the wells is lower. As can be seen in the graph, the pseudo-potential matches well with the full harmonic potential well but only for a certain region. Therefore the transferability to pseudo-potential is limited by the distances between the wells and the constant potential value. 

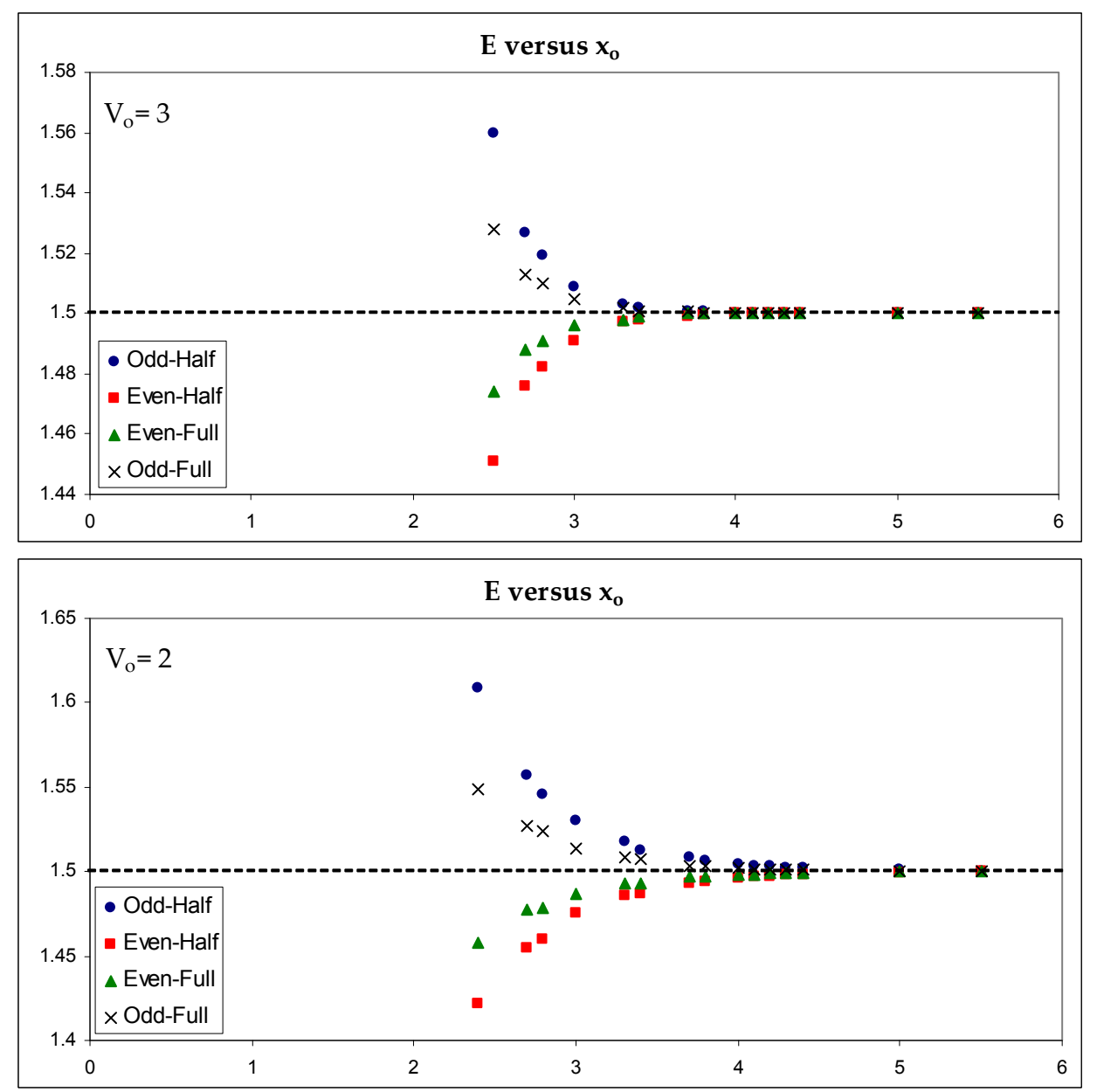

Figure 4.3. The two graphs shows that the transferability improves when $V_{o}$ increases and for large values of $x_{0}$.

\section{$\underline{\text { Pseudo-potential for alkali atoms }}$}

Before I analyze the alkali metals I need to consider the properties of the individual alkali atoms. All the alkali atoms have one electron in the valence level, which is the main component for the atom. The rest of the electrons are in closed shells and require more energy to remove. This closed shell region can be considered the core region of the atom and is unaffected when outside sources interact with the atom. In 
order to ignore the energy of this region, a pseudo potential must be produced. This effective potential will be used to study the properties of alkali metal.

To construct a suitable pseudo-potential I need to use a program and see whether the energy is comparable to the valence energy from an all-electron calculation of the atom. Certain input parameters are required for the program which will generate values of the pseudo-potential at different radial distances. I need to indicate which element to use. From there, I need to indicate what level of the electronic configuration is considered the boundary of the core region. Then, I input the number of valence levels and a particular set of principal and azimuthal quantum numbers. For instance, if I were to use Sodium $(\mathrm{Na})$, I can choose three valence levels $3 \mathrm{~s}, 3 \mathrm{p}$, and $3 \mathrm{~d}$. Next, I put a fractional amount of an electron (or electrons if there is more than one in the valence region) in each valence level. If the valence levels are $3 s, 3 p$, and $4 s$, I can input $0.7,0.2$, and 0.1, respectively, for the one electron. For this specific setting, the program will first output results that pertain to all the electrons of the atom. These results will contain eigenvalues for each state and properties for each single electron wavefunction. In order for the program to generate a pseudo potential, I need to find an appropriate cut-off by examining the wavefunction of the last state from the all-electron calculation. The cut-off is chosen to be in between where the wavefunction has a node farthest from the origin and the last peak value, which is depicted in figure 4.4.

I generated multiple pseudo-potentials and tested the results by inputting different mixtures of the electrons in the valence levels. Then I compared the energy of 
the electron to the energy of the valence electron in the all-electron calculation. This will indicate which pseudo-potential is a suitable fit. Table 4.1 shows results for the Na atom. I used three valence levels for the electron, 3s, 3p, and 3d. Figure 4.5 shows that trial 10 has a good linear fit. Additional tests were completed for the other alkali atoms. These tests allow me to collect pseudo-potentials for the alkali element, which is necessary to calculate the interaction energy in the system. The next chapter shows the pseudopotentials for the alkali elements.

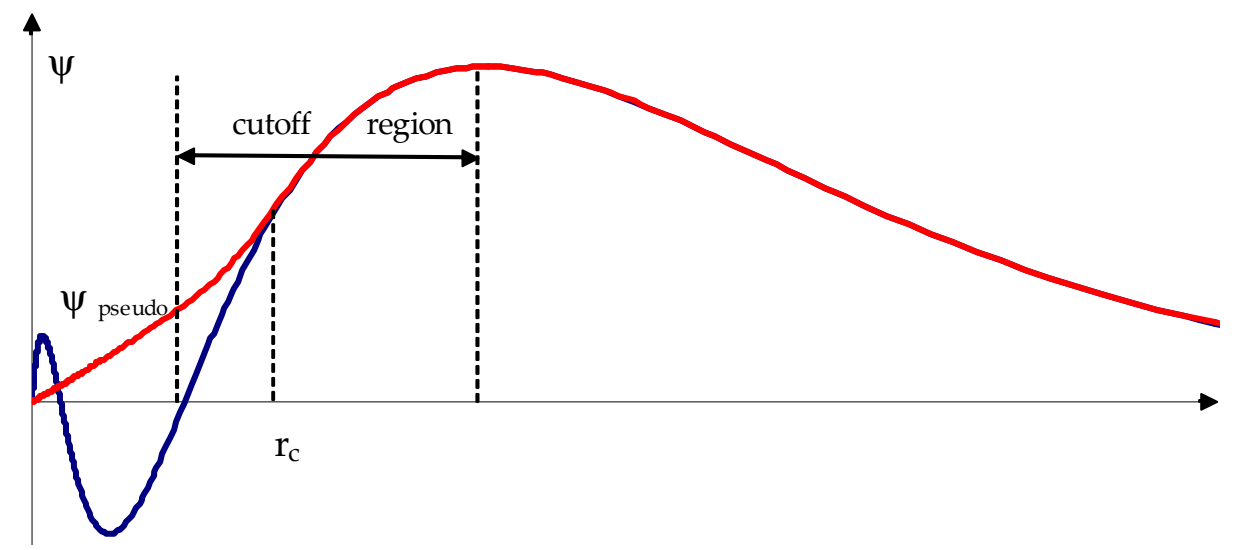

Figure 4.4. Shows the selection of the cut-off region in the wavefunction from the all electron calculation.

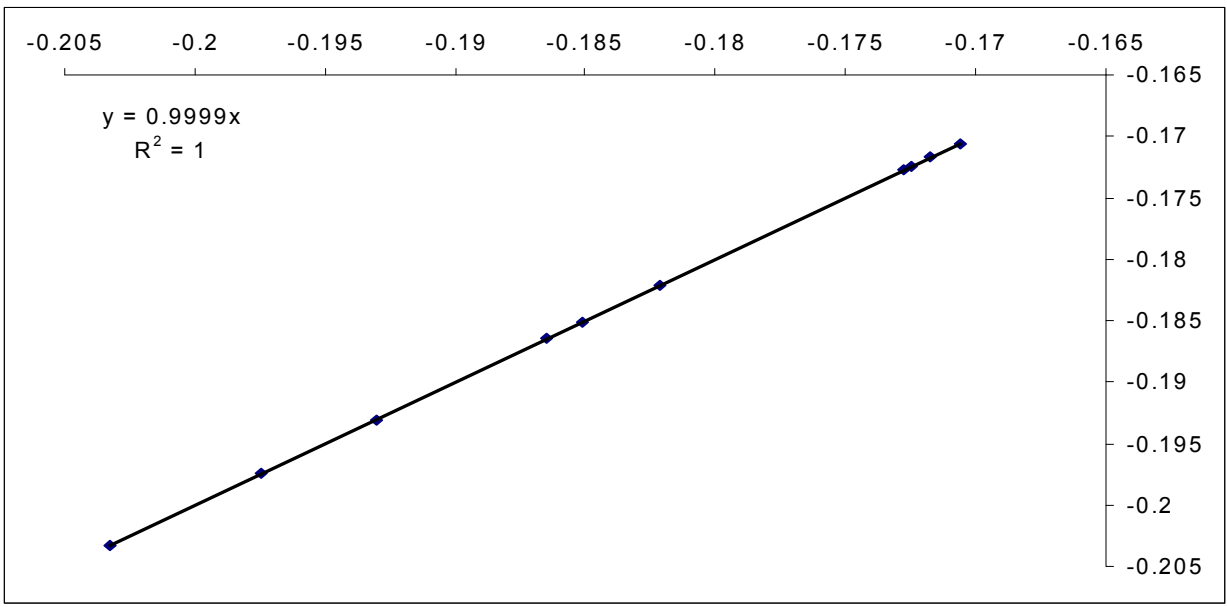

Figure 4.5. Plot of the total energy of trial 10 against the valence energy from the all electron calculation. 


\begin{tabular}{|c|c|c|c|c|c|c|c|c|c|c|c|}
\hline \multirow[b]{3}{*}{$\begin{array}{c}\text { Fraction of electron for the } \\
\text { valence levels } 3 s, 3 p, 3 d\end{array}$} & \multirow[b]{3}{*}{$\begin{array}{l}\text { Valence energy from all } \\
\text { electron calculation }\end{array}$} & & & & & & & & & & \\
\hline & & & & & & Pseudo-pc & tential tes & & & & \\
\hline & & Trial 1 & Trial 2 & Trial 3 & Trial 4 & Trial 5 & Trial 6 & Trial 7 & Trial 8 & Trial 9 & Trial 10 \\
\hline $0.8,0.1,0.1$ & -0.20328 & -0.20221 & -0.20326 & -0.20321 & -0.20322 & -0.20322 & -0.20323 & -0.20327 & -0.20327 & -0.20327 & -0.20328 \\
\hline $0.7,0.2,0.1$ & -0.19306 & -0.18044 & -0.19289 & -0.19300 & -0.19300 & -0.19300 & -0.19301 & -0.19304 & -0.19304 & -0.19303 & -0.19305 \\
\hline $0.7,0.1,0.2$ & -0.19749 & -0.17502 & -0.19341 & -0.19743 & -0.19743 & -0.19737 & -0.19743 & -0.19747 & -0.19746 & -0.19746 & -0.19747 \\
\hline $0.6,0.2,0.2$ & -0.18510 & -0.16139 & -0.17372 & -0.18504 & -0.18504 & -0.18489 & -0.18505 & -0.18507 & -0.18506 & -0.18505 & -0.18507 \\
\hline $0.6,0.3,0.1$ & -0.18211 & -0.15934 & -0.17844 & -0.18206 & -0.18206 & -0.18203 & -0.18206 & -0.18208 & -0.18208 & -0.18207 & -0.18209 \\
\hline $0.6,0.1,0.3$ & -0.18647 & -0.15742 & -0.16887 & -0.18641 & -0.18641 & -0.18602 & -0.18641 & -0.18643 & -0.18643 & -0.18640 & -0.18643 \\
\hline $0.5,0.2,0.3$ & -0.17280 & -0.14282 & -0.15022 & -0.17276 & -0.17275 & -0.17208 & -0.17275 & -0.17275 & -0.17275 & -0.17270 & -0.17276 \\
\hline $0.5,0.3,0.2$ & -0.17249 & -0.13903 & -0.15443 & -0.17244 & -0.17244 & -0.17214 & -0.17244 & -0.17244 & -0.17244 & -0.17241 & -0.17245 \\
\hline $0.5,0.4,0.1$ & -0.1706 & -0.1431 & -0.1582 & -0.1705 & -0.1705 & -0.1704 & -0.1705 & -0.1706 & -0.1705 & -0.1705 & -0.1706 \\
\hline $0.5,0.1,0.4$ & -0.1718 & -0.1392 & -0.1451 & -0.1717 & -0.1717 & -0.1705 & -0.1717 & -0.1717 & -0.1717 & -0.1716 & -0.1717 \\
\hline
\end{tabular}

Table 4.1. Several trials for a pseudo-potential for Sodium (Na) atom. The energy of the pseudo-potential system must be compared to the all electron system to find a proper fit. 


\section{Chapter 5 Results for Alkali Metals}

In the previous three chapters, I discussed various methods to solve the Schrödinger's equation for a multi-electron system. I also discussed how to construct pseudo-potentials to remove particular parts of the system that are insignificant. Each of these tactics has limitations and is usually a too large of an approximation to fully satisfy a system. However, these methods should not be completely ignored. In this chapter, I will show how these methods are supplemental to my calculations for alkali metals.

As I showed in the Jellium model section, the electrons interact with a uniform positive charge disbursed throughout space. This system is solvable and it is able to generate a minimum for the total energy per atom. However, the problem with this system is that it did not match with the minima found for alkali metals, which have different $r_{s}$ values. In order to correct this, the structural ion cannot be ignored. For my model, I will include the positive charges from the ions throughout space in the Jellium model. These ions are considered to be discrete charges. To have the system neutral in charges, I will also include a negative uniform charge distribution throughout space. This will give a total of 4 different charge distributions, which interact with each other. The system can have an imbalance of charge because of the ions and electrons but the uniform negative background has equal and opposite amount of charge compared to the uniform positive background. I will now have a total of 10 interaction energy terms instead of 3 terms as in the Jellium model. They are $U_{\text {ele-ele, }} U_{\text {ele- } \rho^{+}}, U_{\rho^{+} \rho^{+}}, U_{\text {ion-ion, }}, U_{\text {ion- } \rho^{-}}, U_{\rho^{-} \rho^{-}}$

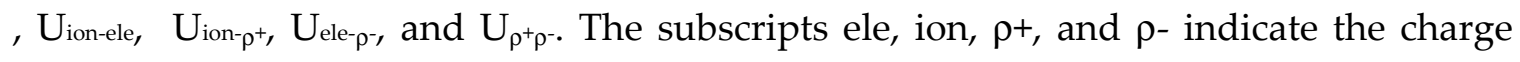


associated with the electron, ionic core, uniform positive charge, and uniform negative charge, respectively. I want to group these terms in such a way that it will give me a group that is identical to the Jellium model. This group will be called the electronic effect and it will contain the kinetic energy term of the electron. A second group will have the ion-ion interaction and I will call it the ionic effect. The rest of the terms that remain will approximate to zero since the charge distribution of $\rho_{+}$and $\rho$ - is the same as the electron, ele. This form is expressed as

$$
\begin{gathered}
\hat{\mathrm{H}}_{\text {eef }}=\mathrm{K}_{\text {ele }}+\mathrm{U}_{\text {ele-ele }}+\mathrm{U}_{\text {ele- } \rho+}+\mathrm{U}_{\rho+\rho+}(\text { electronic effect }), \\
\left.\mathrm{E}_{\text {ief }}=\mathrm{U}_{\text {ion-ion }}+\mathrm{U}_{\text {ion- } \rho-}+\mathrm{U}_{\rho-\rho-} \text { (ionic effect }\right), \\
\mathrm{U}_{\text {ion-ele }}+\mathrm{U}_{\text {ion- } \rho+} \cong 0 \\
\mathrm{U}_{\text {ele- } \rho-}+\mathrm{U}_{\rho+\rho-} \cong 0 .
\end{gathered}
$$

The electronic effect will be solved through Quantum Mechanics while the ionic effect will be solved Classically. The first term in the ionic effect expression is calculated by Ewald summation [21]. The calculation involves summing the long-range interaction in Fourier space and the short-range interaction in real space. Depending on the lattice structure, $\mathrm{U}_{\text {ion-ion }}$ will have different results. For alkali metals, the lattice type is bodycentered cubic (bcc). Each of the terms in the ionic effect diverges individually as the system increases, however, the sum of the 3 terms will converge to a constant value for a fixed $r_{s}$. Uion-p- will differ for each element when $\rho$ - interacts with the core region of the ion. This core region has a different electric potential for each element. To describe the electric potential of the ions, I will generate pseudo potentials with the program that was explained in the previous chapter for alkali atoms. The pseudo-potential from the ion is 


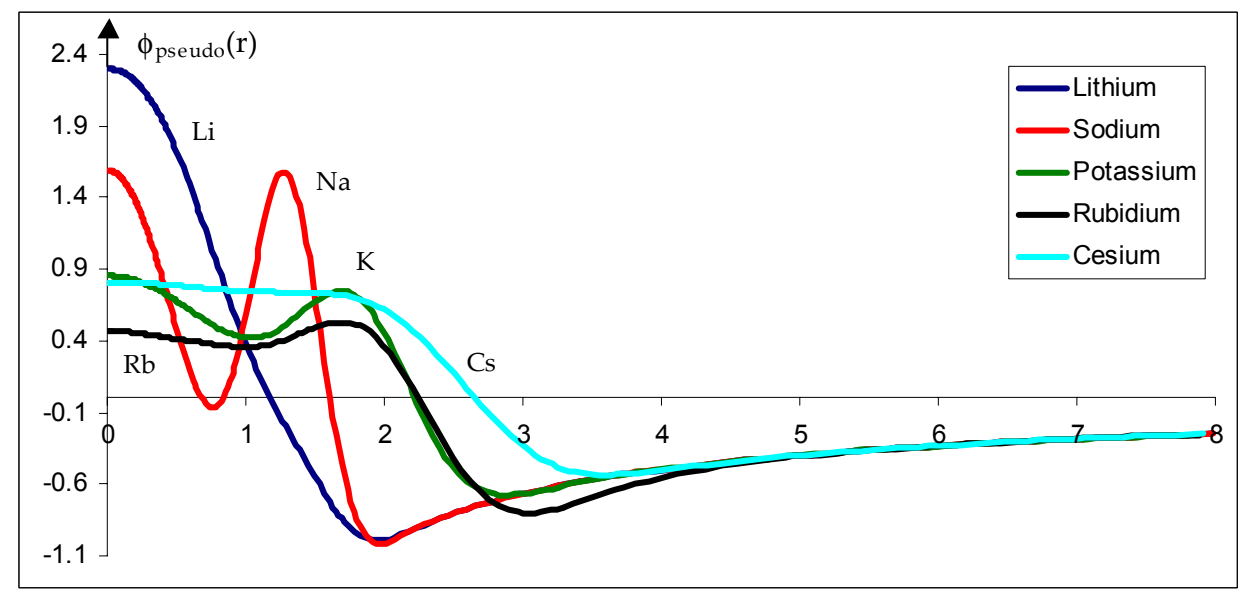

Figure 5.1. Pseudo-potentials (Ry/charge) for alkali atoms versus $r\left(\mathrm{a}_{\mathrm{o}}\right)$. These are used to calculate the interaction energy.

used to calculate the potential energy between the ion and any charge that interacts with it. The generated pseudo-potential for alkali atoms is shown in figure 5.1. If I ignore the ion having a core and treat it as a positive point charge, the ionic effect will not change for different elements. The ionic effect energy for bulk will be $-1.7918585 / \mathrm{r}_{\mathrm{s}}$ for a bcc lattice type and $-1.7917525 / \mathrm{r}_{\mathrm{s}}$ for fcc (face-centered cubic). These results also show that bcc is favored since it is lower in energy than fcc, which is in agreement with experiments for alkali metals. With this ionic effect energy, I can correct it by removing the interaction energy between the positive point charge and $\rho$ - within the core region, and add the energy due to the pseudo-potential of the ion and $\rho$-from the core region. This correction is accomplished computationally. Table 5.1 shows the ionic effect energy for alkali metals at different $r_{s}$ values. Adding this ionic effect to the Jellium model (the electronic effect) adjusts the $r_{s}$ minimum. As $I$ showed in figure $3.1, r_{s} / a_{\circ}$ has a minimum at 3.83 for the Jellium model. 


\begin{tabular}{|c|c|c|c|c|c|}
\hline \multirow[b]{2}{*}{$\mathbf{r}_{\mathrm{s}} / \mathbf{a}_{\mathrm{o}}$} & \multicolumn{5}{|c|}{ Ionic effect } \\
\hline & $\mathbf{L i}$ & $\mathrm{Na}$ & $\mathrm{K}$ & $\mathbf{R b}$ & Cs \\
\hline 8.594 & -0.195 & -0.188 & -0.177 & -0.175 & -0.168 \\
\hline 8.141 & -0.204 & -0.195 & -0.183 & -0.180 & -0.172 \\
\hline 7.734 & -0.213 & -0.203 & -0.188 & -0.185 & -0.176 \\
\hline 7.366 & -0.222 & -0.210 & -0.193 & -0.190 & -0.179 \\
\hline 7.031 & -0.230 & -0.217 & -0.197 & -0.193 & -0.180 \\
\hline 6.725 & -0.238 & -0.223 & -0.201 & -0.196 & -0.181 \\
\hline 6.445 & -0.246 & -0.228 & -0.203 & -0.198 & -0.181 \\
\hline 6.187 & -0.254 & -0.234 & -0.205 & -0.199 & -0.180 \\
\hline 6.066 & -0.257 & -0.236 & -0.206 & -0.199 & -0.180 \\
\hline 5.837 & -0.264 & -0.240 & -0.206 & -0.199 & -0.177 \\
\hline 5.729 & -0.267 & -0.242 & -0.206 & -0.199 & -0.175 \\
\hline 5.524 & -0.274 & -0.246 & -0.206 & -0.197 & -0.171 \\
\hline 5.334 & -0.280 & -0.248 & -0.204 & -0.195 & -0.166 \\
\hline 5.156 & -0.285 & -0.251 & -0.202 & -0.191 & -0.159 \\
\hline 4.990 & -0.290 & -0.252 & -0.198 & -0.187 & -0.151 \\
\hline 4.834 & -0.295 & -0.253 & -0.194 & -0.181 & -0.142 \\
\hline 4.687 & -0.299 & -0.253 & -0.188 & -0.174 & -0.131 \\
\hline 4.550 & -0.303 & -0.253 & -0.182 & -0.166 & -0.119 \\
\hline 4.420 & -0.307 & -0.252 & -0.174 & -0.157 & -0.106 \\
\hline 4.297 & -0.309 & -0.250 & -0.165 & -0.147 & -0.091 \\
\hline 4.238 & -0.311 & -0.248 & -0.160 & -0.141 & -0.083 \\
\hline 4.181 & -0.312 & -0.247 & -0.155 & -0.135 & -0.075 \\
\hline 4.125 & -0.313 & -0.245 & -0.150 & -0.129 & -0.066 \\
\hline 4.018 & -0.314 & -0.241 & -0.138 & -0.116 & -0.047 \\
\hline 3.966 & -0.315 & -0.239 & -0.131 & -0.108 & -0.037 \\
\hline 3.916 & -0.315 & -0.236 & -0.125 & -0.101 & -0.027 \\
\hline 3.867 & -0.316 & -0.234 & -0.118 & -0.093 & -0.016 \\
\hline 3.819 & -0.316 & -0.231 & -0.110 & -0.085 & -0.005 \\
\hline 3.773 & -0.316 & -0.227 & -0.103 & -0.076 & 0.006 \\
\hline 3.683 & -0.316 & -0.221 & -0.086 & -0.058 & 0.031 \\
\hline 3.516 & -0.313 & -0.204 & -0.050 & -0.017 & 0.085 \\
\hline 3.400 & -0.310 & -0.189 & -0.018 & 0.018 & 0.131 \\
\hline 3.300 & -0.305 & -0.173 & 0.013 & 0.053 & 0.176 \\
\hline 3.200 & -0.299 & -0.154 & 0.050 & 0.094 & 0.229 \\
\hline 3.100 & -0.291 & -0.132 & 0.093 & 0.141 & 0.290 \\
\hline 3.000 & -0.281 & -0.105 & 0.143 & 0.196 & 0.360 \\
\hline
\end{tabular}

Table 5.1. The ionic effect energy (Ry/atom) of alkali metals for different $\mathrm{r}_{\mathrm{s}} /$ ao values. 


\begin{tabular}{ccccccc}
\hline $\mathbf{r}$ s/ao & $\mathbf{L i}$ & $\mathbf{N a}$ & $\mathbf{K}$ & $\mathbf{R b}$ & $\mathbf{C s}$ & Jellium \\
\hline Experimental & 3.25 & 3.93 & 4.86 & 5.20 & 5.63 & \\
Theoretical & 3.80 & 4.42 & 5.16 & 5.33 & 5.77 & 3.83 \\
\hline
\end{tabular}

Table 5.2. Minimum $\mathrm{r}_{\mathrm{s}} / \mathrm{a}_{\mathrm{o}}$ for alkali metals. The Jellium is shown as a reference point.

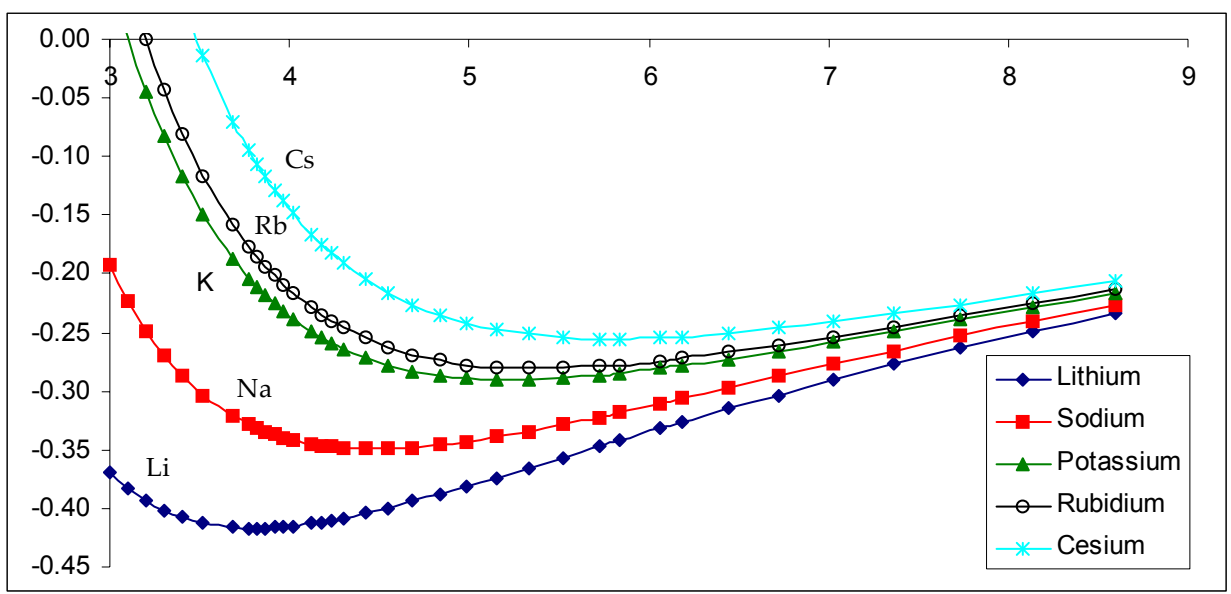

Figure 5.2. Total energy (Ry/atom) versus $\mathrm{r}_{\mathrm{s}} / \mathrm{a}$ o for alkali metals.

When including the ionic effect, the results begin to show a shift of the minimum towards the experimental value, which gives good indication that this approach is valid. The values are shown in table 5.2, and graphically in Figure 5.2, for alkali metals. The theoretical values for the lighter alkali elements have a slightly larger discrepancy than the heavier alkali elements. That is because I assumed the electrons to be free in the system. However, the electrons for the lighter elements seem to not behave in that manner since the core of the ion is smaller, which gives less screening effects. The last two groups from equation 5.1 will not approximate to zero and the electronic effect will be altered since the electrons will not be uniform throughout the system. 


\begin{tabular}{ccccccc}
\hline $\mathbf{B}\left(10^{11} \mathrm{~N} / \mathrm{m}^{2}\right)$ & $\mathbf{L i}$ & $\mathbf{N a}$ & $\mathbf{K}$ & $\mathbf{R b}$ & $\mathbf{C s}$ & Jellium \\
\hline Experimental & 0.116 & 0.068 & 0.032 & 0.031 & 0.020 & \\
Theoretical & 0.089 & 0.056 & 0.032 & 0.027 & 0.020 & 0.028 \\
\hline
\end{tabular}

Table 5.3. Bulk modulus for alkali metals. The value for Jellium is also calculated at the equilibrium.

Another property that can be determined from this system is the bulk modulus.

The relationship for bulk modulus is defined as

$$
B=-V \frac{d P}{d V}=V \frac{d^{2} E}{d V^{2}}
$$

where $\mathrm{P}$ is the pressure applied to the system and V is the volume of the system that the pressure is being applied to. In terms of energy and $\mathrm{r}_{\mathrm{s}} / \mathrm{a}_{\mathrm{o}}$, the bulk modulus at the equilibrium, $\mathrm{P}=0$, is expressed as

$$
\mathrm{B}=\frac{1}{12 \pi \mathrm{a}_{\mathrm{o}}^{3}\left(\mathrm{r}_{\mathrm{s}} / \mathrm{a}_{\mathrm{o}}\right)} \frac{\mathrm{d}^{2} \mathrm{E}}{\mathrm{d}\left(\mathrm{r}_{\mathrm{s}} / \mathrm{a}_{\mathrm{o}}\right)^{2}}
$$

Table 5.3 compares these results with experimental results for alkali metals, which shows good agreement. Again, the lighter elements have a slightly larger discrepancy. The calculation for the bulk modulus is sensitive to the data since it relies on the curvature of the data.

This new scheme shows significant improvement compared to the Jellium model. One reason that the Jellium model could not replicate correctly a real system is the result to the fact that it does not consider the lattice structure. It also showed no change when the system used a different element. That is because the Jellium model assumes uniform charge density throughout the system. Through this new method, I 
was able to demonstrate change to the system when the system was used to analyse a different element and when it incorporated the lattice structure. This new jellium model approach may also be applied to other elements that are not alkali atoms; however, other properties must be factored in to get better results. 


\section{Chapter 6 Conclusion}

After observing the results for alkali metals, one can understand how to apply this new method to smaller systems. The results are essentially composed of the electronic effect and the ionic effect since I considered 4 charge distributions that interact with each other in the system and produced 4 interacting terms that approximate to zero. The electronic effect was calculated by using the Jellium model while the ionic effect involved an electrostatic summation for an infinite system. For a cluster-size system, these two effects will now have boundary constraints. To include this boundary constraint for the electronic effect I can simply apply the Jellium sphere that was discussed in the Jellium model chapter. For the ionic effect, the electrostatic summation will truncate depending on how many atoms will be in the system. This will modify the results of the Jellium sphere. One will discover that the energy of the system will oscillate differently and have different magic numbers for a given element (or $\mathrm{r}_{\mathrm{s}}$ fix value) as the number of atoms increases in the cluster. This could improve the results on describing the stability of certain cluster size since the spherical shell model overestimated the energy difference as was explained for $r_{s}$ fix $=2.0 a_{\circ}$.

A Jellium sphere is just one of the various geometric shapes that can be applied to the electronic effect when analyzing nano-clusters. As I discussed in the first chapter, clusters can have different constructions depending on the size of the cluster and the type of material that composes it. For instance, icosahedron shapes have been observed for small clusters and decahedra for intermediate size clusters of numerous elements. 
Therefore altering the boundary of the Jellium can improve the results for clusters. However, the shape can depend on the material, so finding the correct interaction energy within the system is an important factor. For this new method I developed, the interaction energy involved 4 columbic charges; two of them are due the neutral atom (one valence electron and one positive core charge) and the other two are background charges of equal and opposite amount. This interaction will behave differently depending on the element that composes the system and will determine the geometric shape of the boundary by selecting the lowest energy from all the possible forms of the boundary. Applying this method can produce fruitful results for cluster systems but the most challenging task is determining how the material interacts in the system. Other materials that are not alkali metals have additional properties that cannot be ignored, and these properties can simply be added to my method. 


\section{List of References}

[1] "Magic Nimbers for Metallic Clusters and the Principle of Maximum Hardness". H. K. Harbola, 1992, Proc. Natl. Acad. Sci. USA, 89, 1036.

[2] "Structure, Dynamics, and Thermodynamics of Clusters: Tales from Topographic Potential Surfaces". D. J. Walse, 1996, Science, 271, 5251.

[3] "Introduction to Quantum Mechanics". D. J. Griffiths, 1995, 2nd edition, Pearson Prentice Hall.

[4] “Atomic and Molecular Clusters". R. L. Johnston, 2002, Taylor \& Francis, London and New York.

[5] "Size-Dependent Icosahedral-to-fcc Structure Change Confirmed in Unsupported Nanometer-Sized Copper Clusters". D. Reinhard, B. D. Hall, P. Berthoud, S. Valkealahti, and R. Monot, 1997, Phys. Rev. Lett. 79, 1459.

[6] "Shells of atoms". T. P. Martin, 1996, Phys. Rep. 273, 199.

[7] "Experimental studies of small particle structures". L. D. Marks, 1994, Rep. Prog. Phys. 57, 603.

[8] "Noncrystalline structure of argon clusters. II. Multilayer icosahedral structure of $\operatorname{Ar}_{N}$ clusters $50<N<750$ ". J. Farges, M. F. de Feraudy, B. Raoult, and G. Torchet, 1986, J. Chem. Phys. 84, 3491.

[9] "Metallic bonding and cluster structure". J. M. Soler, M. R. Beltrán, K. Michaelian, I. L. Garzón, P. Ordejón, D. Sánchez-Portal, and E. Artacho, 2000, Phys. Rev. B 61, 5771.

[10] "Electronic Shell Structure and Abundances of Sodium Clusters". W. D. Knight, K. Clemenger, W. A. de Heer, W. A. Saunders, M. Y. Chou, and M. L. Cohen, 1984, Phys. Rev. Lett. 52, 2141.

[11] "Structure and properties of small sodium clusters". I. A. Solov'yov, A. V. Solov'yov, and W. Greiner, 2002, Phys. Rev. A 65, 053203.

[12] "Quantum chemistry of small clusters of elements of groups Ia, Ib, and IIa: fundamental concepts, predictions, and interpretation of experiments". V. BonacicKoutecky, P. Fantucci, and J. Koutecký, 1991, Chem. Rev. 91, 1035. 
[13] "Density-Functional Calculations on Platinum Nanoclusters: $\mathrm{Pt}_{13}, \mathrm{Pt}_{38}$, and Pt55". A. Fortunelli and E. Aprà, 2003, J. Phys. Chem. 107, 2934.

[14] "Silicon Clusters of Intermediate Size: Energetics, Dynamics, and Thermal Effects". L. Mitás, J. C. Grossman, I. Stich, and J. Tobik, 2000, Phys. Rev. Lett. 84, 1479.

[15] "Self-Consistent Field Approach to the Many-Electron Problems". H. Ehrenreich and M. H. Cohen, 1959, Phys. Rev. 115, 786.

[16] "The Configuration Interaction Method: Advances in Highly Correlated Approaches". C. D. Sherrill, H. F. Schaefer, 1999, Advances in Quantum Chemistry, 34, 143.

[17] “Density Functional Theory - an Introduction”. N. Argaman and G. Makov, 1999, arXiv:physics/9806013v2.

[18] “Quantum Theory of Solids". C. Kittel, 1987, Wiley.

[19] "Correlation Energy of an Electron Gas at High Density". M. Gell-Mann and K. A. Brueckner, 1957, Phys. Rev. 106, 364.

[20] "General Theory of Pseudopotentials". B. J, Austin, V. Heine, and L. J. Sham, 1962, Phys. Rev. 127, 276.

[21] “Generalized Ewald Potential Problem”. W. E. Rudge, 1969, Phys. Rev. 181, 1020. 